\title{
A narrative review of research progress on drug therapies for glioblastoma multiforme
}

\author{
Xiangjin Zheng ${ }^{1,2}$, Qin Tang ${ }^{1,2}$, Liwen Ren ${ }^{1,2}$, Jinyi Liu ${ }^{1,2}$, Wan $\mathrm{Li}^{1,2}$, Weiqi Fu ${ }^{1,2}$, Jinhua Wang ${ }^{1,2}$, \\ Guanhua Du, ${ }^{1,2}$
}

${ }^{1}$ The State Key Laboratory of Bioactive Substance and Function of Natural Medicines, Beijing, China; ${ }^{2}$ Key Laboratory of Drug Target Research and Drug Screen, Institute of Materia Medica, Chinese Academy of Medical Science and Peking Union Medical College, Beijing, China

Contributions: (I) Conception and design: X Zheng, Q Tang, J Wang; (II) Administrative support: J Wang, G Du; (III) Provision of study materials or patients: None; (IV) Collection and assembly of data: All authors; (V) Data analysis and interpretation: All authors; (VI) Manuscript writing: All authors; (VII) Final approval of manuscript: All authors.

Correspondence to: Jinhua Wang; Guanhua Du. Institute of Materia Medica, Chinese Academy of Medical Science and Peking Union Medical College, Beijing, China. Email: wjh@imm.ac.cn; dugh@imm.ac.cn.

\begin{abstract}
Glioblastoma multiforme (GBM) is the most aggressive, common, and lethal subtype of malignant gliomas originating from the central nervous system. Currently, the standard therapy for GBM is surgical resection combined with radiation and temozolomide (TMZ). However, the treatment only improves the 2-year survival rate from $10 \%$ to $26 \%$, accompanied by more than $90 \%$ recurrence of GBM tumors at the original site. Low survival rate, serious side effects, and poor prognosis force people to find new therapies. Recent years, the combination of clinical drugs improves the survival rate of GBM patients, but new therapeutic drugs with high-efficiency and low-toxicity are still needed to be discovered. The successful use of immunotherapy in tumor brings hope for people to explore new methods in treating GBM. While the inability to cross the blood-brain barrier (BBB), loss of lymphatic tissue drainage, and antigenpresenting cells in the central nervous system are major reasons for the failure of immunotherapy in the treatment of GBM. Glioma stem cells (GSCs) is a subtype of tumorigenic stem cells which has more specific tumorigenic potential indicating targeting GSCs may be expected to improve therapeutic efficacy. In this review, we discuss clinical drugs that have benefited patients with GBM, cancer immunotherapy for GBM, summarize new drug targets of GBM, and review strategies for increasing the passage of drugs through the BBB.
\end{abstract}

Keywords: Glioblastoma multiforme (GBM); temozolomide (TMZ); bevacizumab; immunotherapy; blood-brain barrier (BBB)

Submitted Dec 16, 2020. Accepted for publication May 07, 2021.

doi: $10.21037 /$ atm-20-8017

View this article at: http://dx.doi.org/10.21037/atm-20-8017

\section{Introduction}

Glioma is the most common primary intracranial neoplasm originating from glial cells, accounting for $32 \%$ of all primary tumors and $81 \%$ of malignant tumors of the central nervous system (1). Glioblastoma multiforme (GBM) is the most malignant (WHO IV grade), aggressive, and frequent subtype, which has its origins in the central nervous system. The incidence of GBM is $46.1 \%$, and it occurs more frequently in males than females (2). The highest occurrence of GBM is in patients aged from 75 to 84 years old, while the median age of newly diagnosed patients is approximately 64 years old. The prognosis of GBM is poor,

^ ORCID: 0000-0001-8367-0787. 

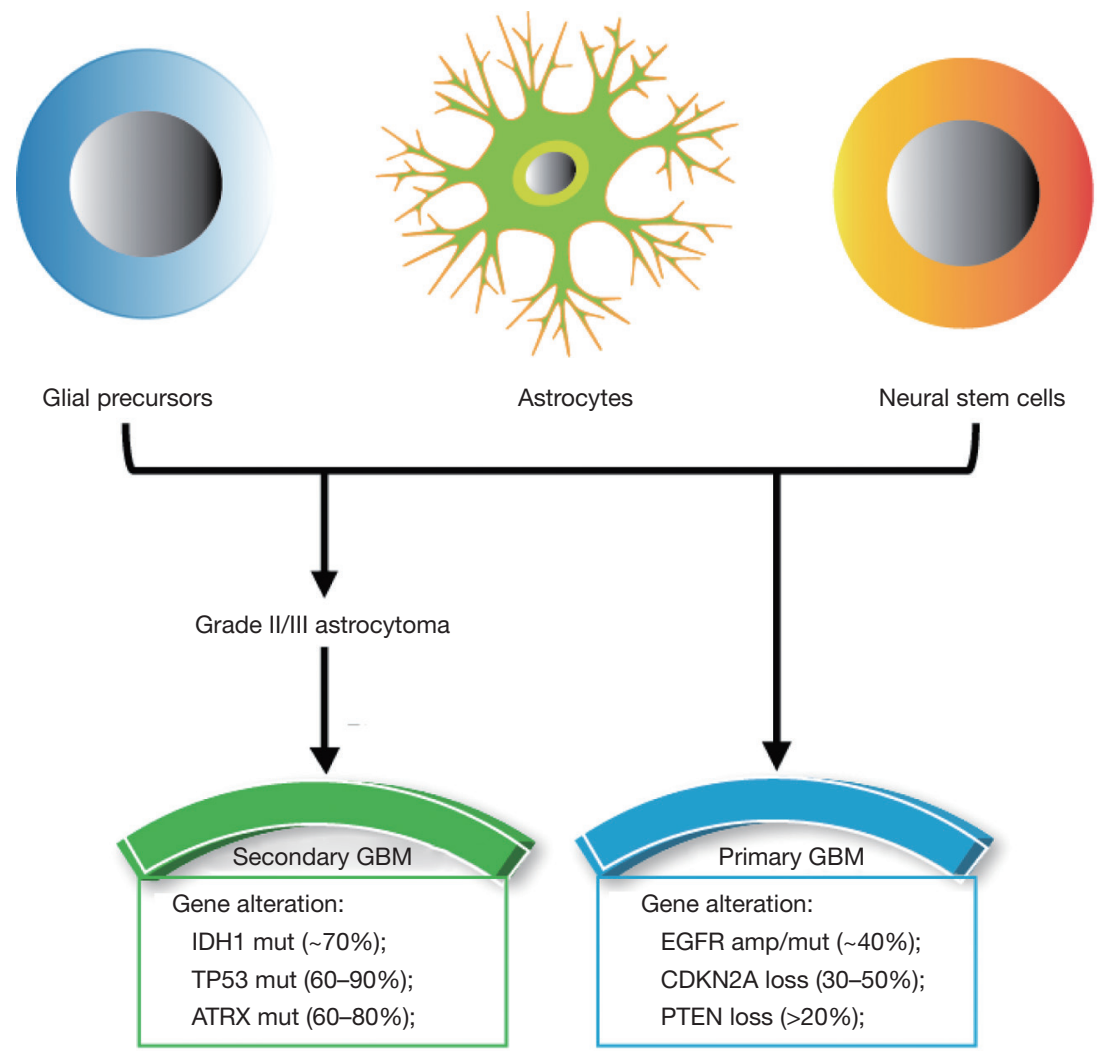

Astrocytes

Neural stem cells

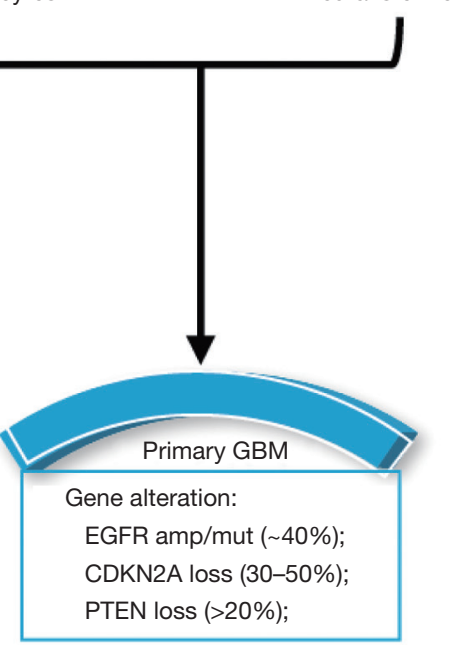

Figure 1 Classification and gene alteration of glioblastoma multiforme (GBM). Glioblastoma multiforme (GBM) originates from glial precursors, astrocytes, and neural stem cells which is divided into primary GBM and secondary GBM. Secondary GBM is transformed from malignant astroglioma at low grades. The genetic mutations of the two kinds of GBM are different. IDH, TP53, and ATRX mutations are more common in secondary GBM than primary GBM. The gene mutations in primary GBM include amplification and mutation of EGFR and CDKN2A and loss of PTEN.

and the median survival time is only $14-17$ months. GBM is prone to recurrence, with a median recurrence time of 6.2 months and a median survival time after a recurrence of only 25-30 weeks. GBM is also the most lethal glioma, with a 1 -year survival rate of approximately $30 \%$ and a 5 -year survival rate of less than $5 \%$ (3).

According to pathological analysis in the clinic, GBM is divided into two categories: primary and secondary. About $95 \%$ of GBM patients have primary cancers, and $5 \%$ have secondary cancers. Most of the patients with secondary GBM are under 45 years old. The pathological difference between the two categories is whether the glioma is low-grade. There is no low-grade glioma in primary GBM, whereas secondary GBM is transformed from malignant astroglioma at low grades. Approximately $70 \%$ of grade II gliomas will develop into grade III/IV gliomas in 5-10 years (4) (Figure 1). Moreover, the genetic mutations of the two kinds of GBM are also different. Isocitrate dehydrogenase 1 (IDH1) is rare in primary GBM (only 20-35\%) but common in most secondary GBMs (about 70\%). Mutations of TP53 (60$90 \%)$ and ATRX (60-80\%) genes occur more frequently in secondary GBM. The gene mutations in primary GBM include amplification and mutation of EGFR (40\%) and CDKN2A $(30-50 \%)$, and loss of PTEN $(>20 \%)(5,6)$ (Figure 1).

To date, the main therapy for GBM is surgery followed by radiation and temozolomide (TMZ). However, it is extremely difficult to safely remove GBM from the brain and completely inhibit its aggressive growth. Also, it is difficult for drugs to pass through the blood-brain barrier $(\mathrm{BBB})$ to reach the tumor site effectively, and even if they do, the pumping system of the BBB will eventually pump drugs out of the brain. Furthermore, the absence of an immune system in the brain leads to the failure of 
immunotherapy for GBM. Currently, the first-line resection treatment only increases the 2-year survival rate from $10 \%$ to $26 \%$ and improves the 6 -year survival rate by only $2 \%$. Although current treatment is far from satisfactory, it does bring hope for GBM patients. In this review, we will introduce some clinical drugs that improve the treatment of GBM, including promising immunotherapies, new therapeutic targets, and strategies to increase the passage of drugs across the BBB.

We present the following article in accordance with the Narrative Review reporting checklist (available at http:// dx.doi.org/10.21037/atm-20-8017).

\section{Drug treatment of GBM in clinical use}

\section{Temozolomide}

In 2005, the European Organization for Research and Treatment of Cancer (EORTC) and the National Cancer Institute of Canada (NCIC) published a surprising result of a clinical trial in which GBM patients aged from 18 to 70 were randomly divided into two groups. Patients in one group were treated with radiotherapy (RT) for 6 months, while patients in the other group were treated with RT combined with TMZ. The results showed that RT treatment combined with TMZ increased the median survival time of GBM patients from 12.1 to 14.6 months and the 2-year survival rate of patients from $10 \%$ to $26 \%$ (7). The first study reported that a combined treatment of RT with TMZ significantly improved the survival rate of patients with GBM, and it was therefore quickly accepted as the standard therapy for GBM treatment and is still used today.

TMZ is an oral methylation reagent that can easily penetrates the BBB with $100 \%$ bioavailability. TMZ can be rapidly absorbed in the brain with a peak plasma concentration time of $0.39-1.33 \mathrm{~h}$. The half-life of TMZ is $1.8 \mathrm{~h}$; it can be excreted quickly and shows no accumulating effect with repeated medication. TMZ is stable under acidic conditions but hydrolyzes into 5-(3-methyl triazene-1-yl) imidazole-4-carboxamide (MTIC) under physiological conditions of $\mathrm{pH}>7$. MTIC is an active metabolite that is unstable and can be further degraded to 5-aminoimidazole4-carboxamide (AIC) and methylated diazo cation $(8,9)$. AIC can be excreted by the kidneys, while methylated diazo cations methylate adenine at $\mathrm{N} 3(9 \%)$, and guanine at O6 (5\%), and N7 (70\%) (10) (Figure 2A). Although the proportion of $\mathrm{O}^{6}$-methylguanine is small, it plays a vital role in the antitumor effect of TMZ. $\mathrm{O}^{6}$-methylguanine cannot prevent replication and transcription of DNA by itself, but it can pair with thymidine during base pairing, thus activating the mismatch repair (MMR) of DNA. MMR recognizes mismatched thymine on the newly synthesized DNA strand and excises it while $\mathrm{O}^{6}$-methylguanine remains on the template strand. Therefore, the ineffective insertionand-clearance cycle of thymidine leads to a continuous break in the DNA strands, which results in the collapse of the replication fork and ultimately induces cell death $(11,12)$ (Figure 2B).

The high expression of $\mathrm{O}^{6}$-methylguanine-DNA methyltransferase (MGMT) and the lack of MMR are the main reasons for GBM to develop resistance to TMZ (13). MGMT can remove the methylation of $\mathrm{O}^{6-}$ methylguanine, restore it to normal guanine, and reduce the toxicity of TMZ without the help of other proteins or cofactors. MGMT binds to the minor groove of DNA containing $\mathrm{O}^{6}$-methylguanine. The targeted $\mathrm{O}^{6}$-methylguanine will be flipped out of the helix and bound to MGMT, and then the $\mathrm{O}^{6}$-methyl group will automatically transfer to the $\mathrm{Cys}^{145}$ residue of MGMT at the active site. The methylated MGMT will lose its activity, become dissociated from DNA, and finally be degraded by the ubiquitin-proteasome system (14). A previous study showed that after MGMT was transfected into SNB19 and U373 GBM cells, the resistance of these two kinds of cells to TMZ was increased 13 times and 5 times, respectively (13).

Attempts were made to inactivate MGMT with the MGMT inhibitor $\mathrm{O}^{6}$-benzylguanine $\left(\mathrm{O}^{6}\right.$ - $\left.\mathrm{BG}\right)$ before treatment with TMZ to overcome TMZ resistance caused by MGMT $(15,16) . \mathrm{O}^{6}-\mathrm{BG}$ is the presumed substrate of MGMT and can deliver its benzyl group to $\mathrm{Cys}^{145}$ of MGMT when it interacts with MGMT, causing irreversible inactivation of MGMT (17). $\mathrm{O}^{6}$-BG cannot be inserted into DNA, but it can directly bind to cytoplasmic and nuclear MGMT (18). Evidence has confirmed that $\mathrm{O}^{6}$ BG significantly enhances the toxicity of TMZ both in vivo and in vitro. However, the phase II trial of TMZ plus $\mathrm{O}^{6}$ benzylguanine in adults with recurrent, TMZ-resistant malignant glioma showed that only 1 in 34 patients with GBM was responsive (15). Subsequently, researchers tried to enhance the efficacy of TMZ by reducing the expression of MGMT. Kim et al. found that the nano-compounds encapsulating wild-type p53 (SGT-53) enhanced the inhibitory effects of TMZ on TMZ-resistant GBM cells both in vitro and in vivo and improved the median survival time of mice transplanted with TMZ-resistant GBM cells (19). 


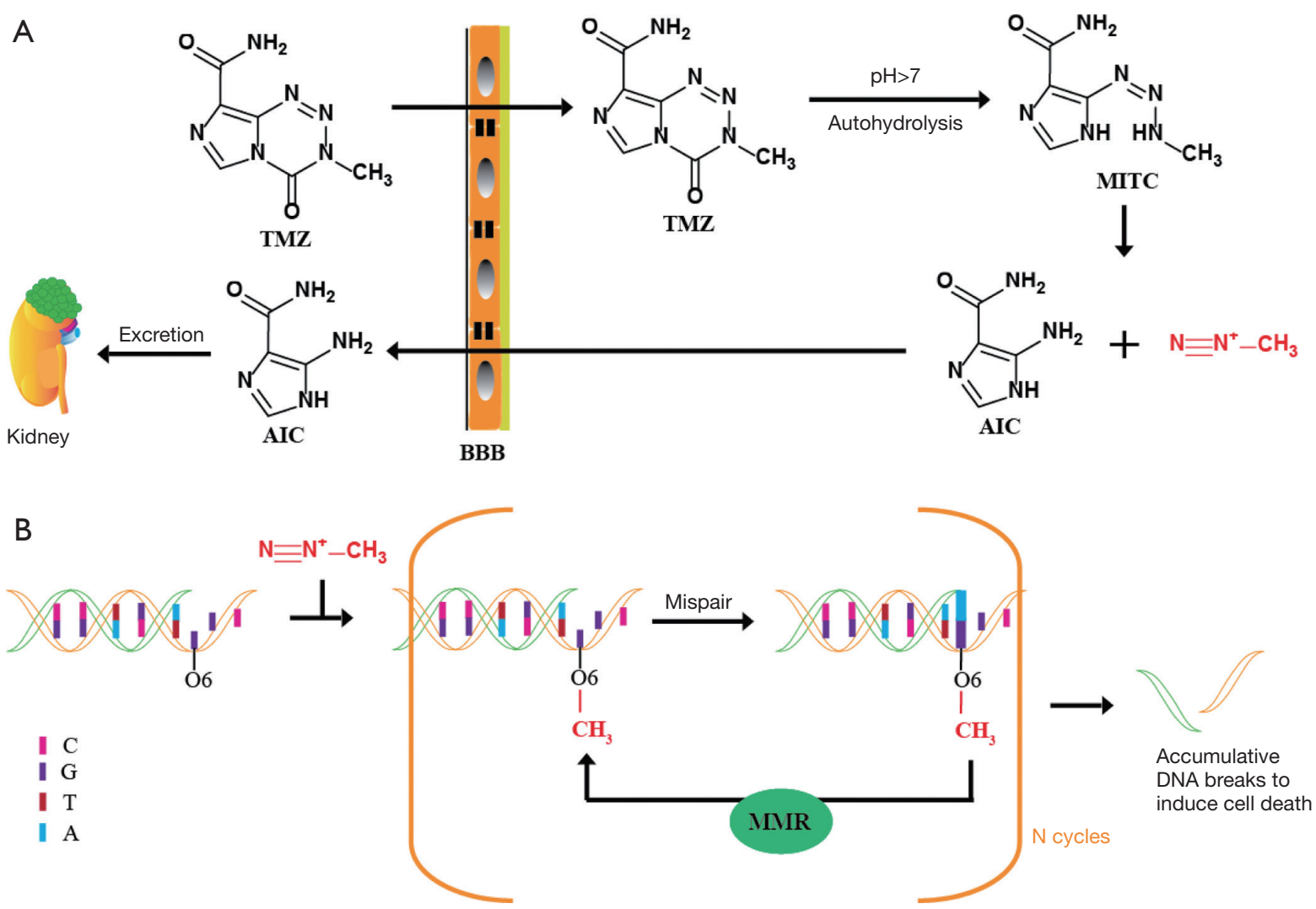

Figure 2 Metabolic pathway and mechanism of Temozolomide (TMZ). (A) TMZ can easily penetrate the BBB with $100 \%$ bioavailability. Then under the physiological conditions of $\mathrm{pH}>7$, TMZ is hydrolyzed into 5-(3-methyl triazene-1-yl) imidazole-4-carboxamide (MTIC). MTIC is an active metabolite that is unstable and can be further degraded to 5-aminoimidazole-4-carboxamide (AIC) and methylated diazo cation. AIC can be excreted by the kidneys, while methylated diazo cations methylate adenine at $\mathrm{N}^{3}$ and guanine at $\mathrm{O}^{6}$ and $\mathrm{N}^{2}$. (B) $\mathrm{O}^{6}-$ methylguanine plays an important role in the antitumor effect of TMZ by leading to the continuous collapse of the replication fork. $\mathrm{O}^{6}$ methylguanine prevents replication and transcription of DNA by pairing with thymidine during base pairing, thus activating the mismatch repair (MMR) of DNA. MMR recognizes mismatched thymine on the newly synthesized DNA strand and excises it while $\mathrm{O}^{6}$-methylguanine remains on the template strand, leading to a continuous break in the DNA strands.

The MGMT gene is located on chromosome 10q26.3, and approximately $60-80 \%$ of gliomas lose chromosome $10 \mathrm{q}$, which results in the low expression of MGMT (12). However, the loss of MGMT activity is mainly attributed to the methylation of the MGMT promoter. This methylation is mainly regulated by 5 '-methylcytosine methyltransferase, which generally occurs on CpG islands in the MGMT gene's promoter region. A previous study showed that the MGMT promoter's methylation inhibited the transcription factor's binding to the MGMT promoter region and ultimately led to the silencing of the MGMT gene (17). Clinical trials have shown that the combination of RT and TMZ has a better effect on patients' methylation of the MGMT promoter (20). Therefore, methylation of the
MGMT promoter can be used as a predictive marker for TMZ chemotherapy. Unfortunately, the positive response only appears in the initial stage of TMZ treatment. In the late stage of treatment, the exposure to $\mathrm{TMZ}$ will result in the loss of MMR function and an increase in TMZ-resistant GBM cells, which will eventually result in the recurrence of GBM, thus highlighting the difficulty of treatment.

In addition to TMZ and RT's combination, researchers have also tried to add other treatments to improve therapeutic efficacy further. Results from several independent clinical trials of bevacizumab, TMZ, and RT in newly diagnosed GBM showed that the addition of bevacizumab prolonged progression-free survival (PFS) with minimal increased toxicity but had little effect on overall 
survival (OS) (21). Tumor-treating fields (TTFields) are antimitotic therapies that mediate the selective disruption of cell division via transduction array by delivering lowdensity, intermediate-frequency $(200 \mathrm{kHz})$ exchange electrons, which in turn induce cell apoptosis during the division phase. In 2015, Stupp et al. published the results of a randomized clinical trial of TTFields combined with TMZ maintenance therapy in GBM patients after chemotherapy. The results showed that, compared with the TMZ group ( $\mathrm{n}=299)$, TTFields combined with TMZ $(\mathrm{n}=466)$ significantly prolonged the PFS (7.1 vs. 4.0 months) and OS (20.5 vs. 15.6 months) of GBM patients, suggesting that TTFields combined with TMZ shows an encouraging prospect for the treatment of GBM (22).

\section{Bevacizumab (BV)}

Angiogenesis is an important sign of the development and metastasis of malignant tumors. GBM is a type of cancer with angiogenesis, and the appearance of new microvascular is a diagnostic marker of GBM. Vascular endothelial growth factor A (VEGF-A), also known as VEGF, is an important regulator of angiogenesis. VEGF promotes the proliferation of vascular endothelial cells through binding to its receptor VEGFR. VEGF is highly expressed in GBM. The inhibition of angiogenesis in GBM by targeting VEGF blocks the nutrient supply and metastatic pathways in the development of GBM and ultimately inhibits the growth and metastasis of GBM (23). Based on this idea, VEGF inhibitors are increasingly used for the treatment of GBM. Bevacizumab (BV) was the first synthetic monoclonal IgG1 antibody to be approved by the Food and Drug Administration (FDA) in 2004 and was approved to treat recurrent GBM by the FDA in March 2009. BV inhibits angiogenesis by binding to VEGF and blocking the binding of VEGF to its receptor. The half-life of BV in humans is 21 days, and the time to reach steady-state is expected to be 100 days. The main adverse effects of BV are cardiovascular and blood system disorders, such as hypertension and thrombosis (24).

Since the FDA approved BV to treat recurrent GBM, researchers have been trying to combine it with other chemotherapy drugs. However, the results were disappointed. The drug that was trialed first with BV was irinotecan, an inhibitor of DNA topoisomerase I. The combined therapy slightly improved the 6-month PFS compared with BV alone (50.3\% vs. $42.6 \%)$ but did not improve OS (8.7 vs. 9.2 months) and increased the adverse effects $(65.8 \%$ vs. 46.4\%) (25). Based on the results from the combined treatment of $\mathrm{BV}$ and irinotecan, the researchers hoped to improve the efficacy by adding a third drug but were unsuccessful. The combined treatment of BV, irinotecan, and cetuximab reduced the 6-month PFS rate and median OS of patients with recurrent GBM to $30 \%$ and 29 weeks, respectively (26). Another study reported that combined BV, irinotecan and carboplatin treatment resulted in a PFS rate and median OS of $46.5 \%$ and 8.3 months, respectively, in patients with naive recurrent GBM (27). Further research efforts were then made to combine sorafenib or fotemustine with $\mathrm{BV}$ in patients with recurrent GBM. However, the former was less effective than BV alone (28), and the latter did not improve efficacy (29).

Lomustine is the only chemotherapy drug that can improve the efficacy of $\mathrm{BV}$ in patients with recurrent GBM. Lomustine can pass through the BBB and exert its antitumor effects by alkylating DNA, RNA, and proteins. Taal et al. published the results of an exciting phase II clinical trial in 2014. The 9-month OS rate of the BV group ( $\mathrm{n}=51)$, lomustine group $(\mathrm{n}=47)$, and $\mathrm{BV}$ combined with the lomustine group was $38 \%, 43 \%$, and $59 \%$, respectively. The median OS was 8 months, 8 months, and 11 months, respectively, and the 6-month PFS rate was $18 \%, 11 \%$, and $41 \%$, respectively. The proportion of side effects higher than level 3 was $36 \%, 20 \%$, and $54 \%$, respectively (30). Although the combination of $\mathrm{BV}$ and lomustine increased toxicity, its efficacy was also significantly increased, suggesting a promising potential for combining these two drugs in the treatment of patients with recurrent GBM. The results of phase III clinical trials are highly anticipated. In 2017, Wick et al. published GBM patients' results with a combination of $\mathrm{BV}$ and lomustine. In the clinical trial, 437 patients were randomly divided into two groups, a combined BV and lomustine group ( $\mathrm{n}=288)$ and a lomustine-alone group $(\mathrm{n}=149)$. After treatment, 329 patients (75.3\%) survived, but the combination group did not show survival advantages. The median OS of the combination group and the lomustine-alone group was 9.1 and 8.6 months, respectively. PFS was slightly longer in the combination group than in the single-use group (4.2 vs. 1.5 months), but the rate of level 3-5 side-effects in the combination group was also higher than that in the singleuse group (63.6\% vs. 38.1\%). Moreover, the combination group did not demonstrate improved quality of life or improved neurocognitive function (31). Therefore, the efficacy of lomustine combined with BV in the treatment of recurrent GBM needs further validation and confirmation. 


\section{Promising immunotherapy}

Since 1998, when the FDA approved the first cytokine (interleukin-2)-based immunotherapy for the treatment of melanoma, immunotherapy has continued to attract researchers' attention, and there have been many breakthroughs in the past 10 years. In 2011, the FDA approved the fully synthetic monoclonal antibody Ipilimumab against CTLA-4 to treat metastatic melanoma. In 2014, the FDA approved the anti-PD-1 antibodies Pembrolizumab and Nivolumab for metastatic melanoma. In 2015, the FDA approved Nivolumab for the treatment of non-small cell lung cancer (32). However, to date, immunotherapy has had little success in the treatment of GBM.

The activation of cytotoxic T cells (CD8+) is completed through two steps: firstly, the T-cell receptor (TCR) binds to the MHC-peptide complex I and recognizes the cancer cells; secondly, TCR binds to the co-stimulatory factors of target cells and fully activates $\mathrm{T}$ cells, thereby promoting the proliferation of $\mathrm{T}$ cells. On the one hand, cancer cells bind to T cells via surface co-repressors (B7-1, CTAL-4, PD-L1, and PD-1, etc.) or secrete immunosuppressive factors (TGF- $\beta$, IL-6, IL-10, etc.) to block the activation and proliferation of T cells. MCH I is expressed at low level, while co-repressor PD-L1 is highly expressed in GBM cells. Also, GBM cells inhibit T cells' activation and proliferation by secreting immunosuppressive factors (TGF- $\beta$, IL-6, IL-10, etc.). On the other hand, the inability to cross the $\mathrm{BBB}$, the loss of lymphatic tissue drainage, and antigen-presenting cells in the CNS are major reasons for the failure of immunotherapy in the treatment of GBM. Despite this, the enormous advantage of immunotherapy in targeting tumor cells without harming normal cells has attracted researchers to explore this therapeutic strategy, and consequently, immunotherapy has made some progress in recent years.

The success of immunotherapy is attributed to the recognition of tumor cells by the immune cells. The important surface biomarkers of GBM are EGFRvIII and IL13Ra2. EGFRvIII is the most common mutation in EGFR and occurs most frequently in GBM (about $30 \%)$ and is associated with low long-term survival in patients (33). EGFRvIII is a novel epitope with tumorspecific and immunogenicity, which is formed by deleting EGFR exon 2-7 and the appearance of a new Gly residue at the junction of 1 and 8. Furthermore, EGFRvIII is not expressed in normal brain tissue (34). Immunotherapy based on EGFRvIII has two types: peptide vaccines and adoptive therapy (Figure 3). Rindopepimut (CDX-110) is a peptide vaccine formed by the keyhole limpet hemocyanin (KLH) binding to the C-terminal of 14 amino acids (NH2Leu-Glu-Glu-Lys-Lys-Gly-Asn-Tyr-Val-Val-Thr-AspHis-Cyt-COOH) at the EGFRvIII mutated location (35) (Figure 3). From 2004 to 2009, three-phase II clinical trials of Rindopepimut (ACTIVATE, ACTII, and ACTIII) were completed, and consistent results were obtained. Compared with EGFRVIII ${ }^{+}$controls, the median OS and PFS were extended to 24.6 months and 15.2 months, respectively. Rindopepimut has the same efficacy for patients with or without MGMT promoter methylation. Rindopepimut is well tolerated and stimulates specific immune responses stably and permanently (36). In 2017, Weller et al. reported the results of a large phase III clinical trial of Rindopepimut. Newly diagnosed GBM patients were randomly divided into two groups; placebo combined with TMZ $(\mathrm{n}=371)$ or Rindopepimut combined with TMZ ( $\mathrm{n}=374)$. There were no differences in OS (median OS 20. 0 vs. 20.1 months, respectively) between the two groups (37). An alternative research approach is to destroy GBM cells by importing $\mathrm{T}$ cells that express chimeric antigen receptors (CARs), targeting EGFRvIII. In humans and mice, the CARs targeting EGFRvIII are primarily based on single-chain variable fragments (scFvs): 139 for humans and 3C10 for mice $(38,39)$. According to the CARs derived from murine $3 \mathrm{C} 10$ scFvs, which successfully inhibited GBM xenografts' growth in vivo, Johnson et al. designed, synthesized, and screened out the CARs of human scFVs139, which restrained the growth of both subcutaneous and orthotopic GBM xenografts (34). Currently, CAR-T cells are being used in phase I clinical trials to treat recurrent GBM (NCT02209376).

IL13R $\alpha 2$ is a monomer with a high affinity for IL-13 (40). IL $13 \mathrm{R} \alpha 2$ is highly expressed in more than $50 \%$ GBM but rarely distributed in normal brain tissues (41). The expression of IL13R $\alpha 2$ is increased as GBM malignancy develops and is independent of the degree of tumor differentiation (42). IL13R $\alpha 2$ is also related to the decreased long-term survival rate of GBM patients (43). Brown et al. developed an IL13Ra2-specific, MHC-independent CAR and named it IL13-zetakine. This CAR recognizes IL13R $\alpha 2$ by IL13 ligands mutating at a single site (E13Y) and exerts its effect through the intracellular $\mathrm{CD} 3 \zeta \mathrm{T}$ cell activation domain (44). It was further confirmed that CAR-T cells inhibited the growth of GBM tumors in a xenograft model. Brown et al. introduced the CAR-T cells into the brains of three patients with recurrent GBM by intracranial 

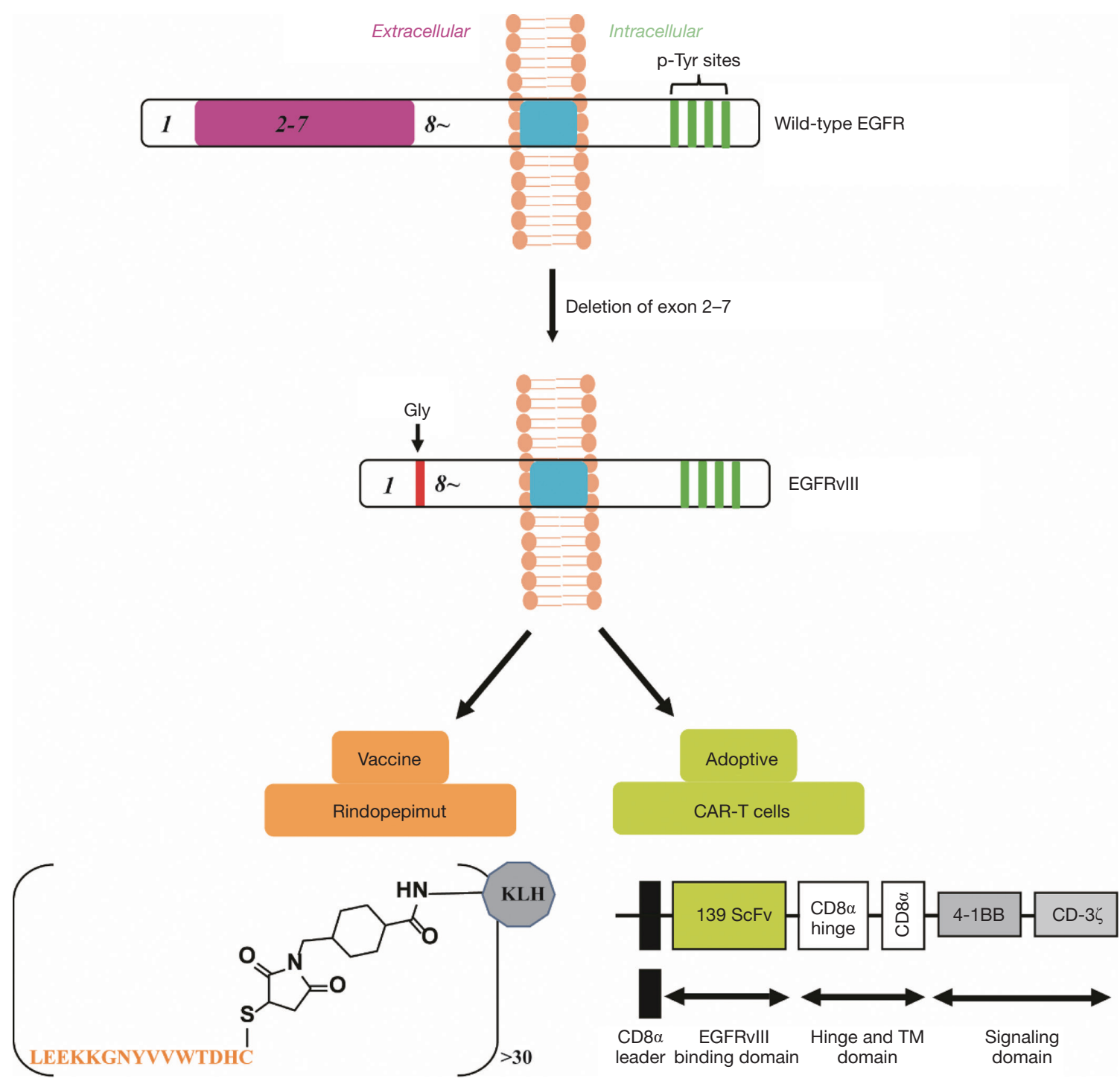

Figure 3 The strategies of immunologic therapies based on EGFRvIII. A EGFRvIII is a common mutation of epidermal growth factor receptor (EGFR), which is formed by deleting EGFR exon 2-7 and the appearance of a new Gly residue at the junction of 1 and 8 . Peptide vaccines and adoptive therapy are the two main immunotherapies based on EGFRvIII. Rindopepimut (CDX-110) is a peptide vaccine formed by the keyhole limpet hemocyanin (KLH) binding to the C-terminal of 14 amino acids (NH2-Leu-Glu-Glu-Lys-Lys-Gly-AsnTyr-Val-Val-Thr-Asp-His-Cyt-COOH) at the EGFRvIII mutated location. Chimeric antigen receptor T cell immunotherapy (CAR-T) is another method targeting EGFRvIII. The CAR targeting EGFRvIII is primarily based on single-chain variable fragments (scFvs). IL13R $\alpha 2$ is highly expressed in GBM. An IL13R $\alpha 2$-specific, MHC-independent CAR recognized IL13R $\alpha 2$ by IL3 ligands mutating at a single site

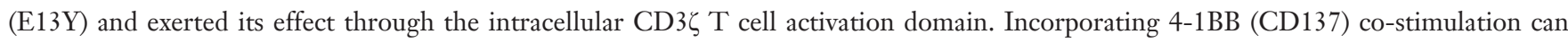
improve antitumor potency by reducing the off-target effect.

injection and found that the CAR-T cells were well tolerated, and patients had a transient and controlled CNS inflammation. However, a larger tumor necrosis area was found at the IL13-zetakine+ T cell injection site (45). Krebs et al. showed that CARs constructed by a single mutant
(E13Y) IL13 ligand recognized not only IL13R $\alpha 2$ but also IL13R $\alpha 1$. IL13R $\alpha 1$ itself has a weak affinity with IL13 but forms a heterodimer with IL4R to bind IL13 under physiological conditions, and heterodimers are abundantly distributed in normal brain tissue. The results of GBM 
orthotopic transplantation in vivo demonstrated that CAR T cells with the IL13 mutation (E13/Y) did not inhibit the growth of the transplanted tumor or improve the survival rate of mice. Only the CAR T cells constructed with IL13 containing the E13K single mutation or E13K and K105R simultaneous mutations killed the transplanted tumor and significantly improved mice's survival rate (46). By using a surface plasmon resonance (SPR) experiment, Van Nguyen et al. identified that simultaneously mutating $\mathrm{Glu}^{13}, \mathrm{Arg}^{66}$, $\mathrm{Ser}^{69}$, and Lys ${ }^{105}$ of IL13 into Lys ${ }^{13}$, Asp ${ }^{66}$, Asp ${ }^{69}$, and Arg ${ }^{105}$ significantly enhanced the affinity of IL13 to IL13R $\alpha 2$ (Kd $\sim 5 \mathrm{nM})$ and did not bind to the heterodimer of IL13R R1/ IL4R. Furthermore, radiolabeled CAR-T cells of IL13 with the four mutated amino acids significantly inhibited the growth of subcutaneous and orthotopic xenografts of IL13R $\alpha 2^{+}$GBM cells and greatly improved the survival cycle of mice (47).

\section{New targeted therapy}

Glioma stem cells (GSCs) are a subtype of tumorigenic stem cells and determine the tumor's heterogeneity. GSCs are characterized by self-renewal cells. GSCs increase the tumor cells, which have more specific tumorigenic potential, and decide the recombinant initial tumor's cloning phenotype during transplantation. Therefore, GSCs are considered as the main drivers of malignancy and progressive GBM. More importantly, GSCs are naturally resistant to chemotherapy and radiation therapy. Moreover, they are more aggressive than normal brain stem cells and can escape from current treatments, which contributes to the tumor's recurrence. Targeting these cells is expected to improve therapeutic efficacy.

Targeting GSCs has mainly focused on two aspects: (I) directly targeting the GSCs; and (II) targeting the microenvironment of the GSCs, namely, the niche. By isolating tumor cells from tissues of high-grade glioma patients, researchers found that GSCs contain two distinct and mutually exclusive subpopulations, proneural $(\mathrm{PN})$ and mesenchymal (MES). GSCs with the MES subtype are more aggressive and resistant to $\mathrm{RT}$ and have a high expression of ALDH1A3 (48-50). Cheng et al. further confirmed that FOXD1, the upstream transcriptional regulator of ALDH1A3, regulated the clonality of GSCs with the MES subtype in vivo and in vitro. Further analysis of clinical samples of high-grade gliomas revealed that the FOXD1ALDH1A3 signal axis was negatively correlated with patients' prognosis (50). Jin et al. indicated that GSCs with the PN subtype activated EZH2, while BMIT1 was highly expressed in GSCs with the MES subtype. GSCs with the PN subtype are more sensitive to EZH2 destruction, while GSCs with the MES subtype are more sensitive to BMI1 inhibition. In vivo xenograft experiments have demonstrated that the combination of EZH2 and BMI1 inhibitors greatly inhibits tumor growth, suggesting that multi-targeted therapy for GBM could overcome the therapeutic resistance caused by tumor heterogeneity (51). Wee et al. found that ABCG2 increased the expression of cell surface markers of GSCs and promoted self-renewal of glioma cells, whereas it did not affect the RT resistance of glioma cells. Yang et al. suggested that histone deacetylase 6 (HDAC6) inhibitors induced the differentiation of GSCs and enhanced the RT resistance of cancer cells via the SHH/Gli1 signaling pathway (52). Therefore, the combination of ABCG2 and HDAC6 inhibitors may exert better therapeutic effects and attenuate RT resistance in GBM. Common biomarkers of GSCs are CD133, CD44, Nestin, and Sox2. Also, other biomarkers of GSCs have been found in recent years, such as S100A4. Knockdown of S100A4 has been found to completely block tumor formation in vivo and in vitro (53).

The niche of GSCs mainly consists of four categories: (I) blood vessels; (II) hypoxia; (III) cancer cell-associated fibroblasts; and (IV) tumor-associated macrophages. Angiogenesis is the typical feature of malignant gliomas, and many studies have focused on it. However, BV is currently the only anti-angiogenesis drug approved by the FDA to treat relapsed GBM. Hypoxia plays an important role in the development of GSCs. Persano et al. revealed that GSCs preferred to stay in the tumor's hypoxic core, while more differentiated tumor cells were mainly located in the peripheral and vascular parts of the tumor $(54,55)$.

When the primary glioma sphere was incubated at a physiological concentration of $7 \%$ oxygen, the expression of CD133 was nearly doubled. Hypoxia (1\%) increased the self-renewal capacity of the CD133(+) human GSCs $(56,57)$. Lee et al. established the recurrence model of GBM by samples derived from patients who received TMZ treatment. In this model, the expression of the hypoxiainducible factors HIF 1a and HIF2a was significantly increased, and they both played important roles in the development of non-stemness glioma cells. Knockoff of HIF1a and HIF2a inhibited the conversion of non-stemness glioma cells to GSCs (58). Stromal fibroblasts promote the formation of the cardiovascular system by secreting VEGF, participating in the initiation of tumor angiogenesis via fibronectin and type I collagen, and recruiting 


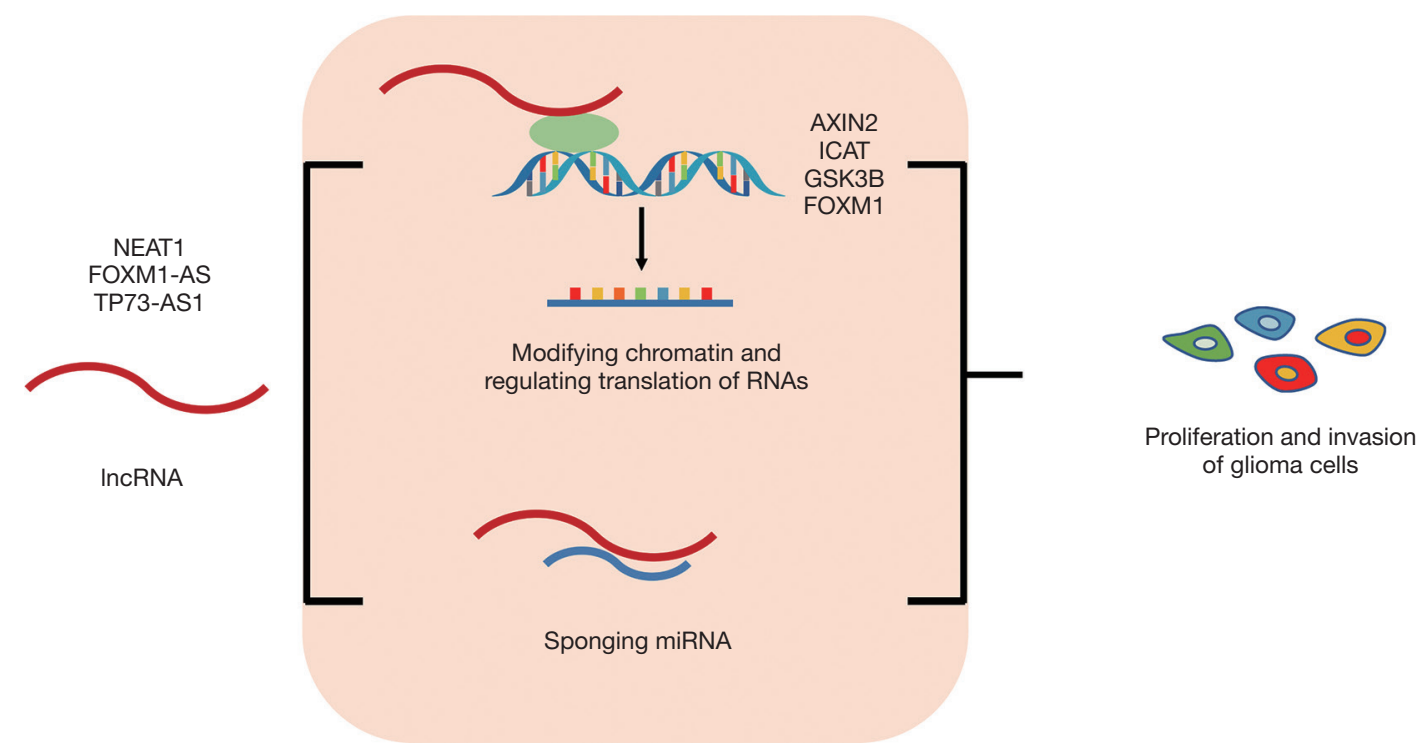

Figure 4 The main functions of long non-coding RNAs (lncRNAs) on glioma progression. Long non-coding RNAs (LncRNA) NEAT1 inhibits AXIN2, ICAT, and GSK3B and up-regulates WNT/ $\beta$-catenin signaling by binding to EZH2, eventually promoting glioma cell growth and invasion. FOXM1-AS can promote proliferation and tumorigenesis of GSCs by promoting the interaction of ALKBH5 with FOXM1. LncRNA TP73-AS1 can sponge miR-142 and promote HMGB1 expression, which contributes to glioma cell proliferation and invasion.

endothelial progenitor cells to the tumor to accelerate angiogenesis and the growth of the tumor through SDF-1 $(59,60)$. Tumor-associated macrophages secrete major inflammatory components of the stroma in various tumors, but their function in GSCs remains unclear. By targeting the regulatory proteins of GSCs in the niche, both tumor stem cells and recurrence of GBM might be reduced, and ultimately the prognosis of patients would be improved.

Long non-coding RNAs (lncRNAs) have recently been identified that might be critical for cancer malignancy and may participate in glioma initiation, progression, and other malignant phenotypes. LncRNAs are a type of RNA which have more than 200 nucleotides without a protein-coding function (61). LncRNAs are important for posttranscriptional regulation, such as chromatin modification (62), translation of RNAs (63), and modulation of alternative splicing (64), and they also act as microRNA sponges (65). Though few lncRNAs have been well characterized, lncRNAs exert significant and precise roles in cancer's biological processes.

LncRNAs play an important role in glioma progression. The role of lncRNAs in glioma progression can be mainly classified into two processes: (I) modifying chromatin and regulating translation of RNAs; and (II) sponging miRNA (Figure 4). Firstly, lncRNAs can modify chromatin and regulate relative gene expression. Chen and colleagues identified NEAT1, a lncRNA that binds to EZH2 and promotes trimethylation of $\mathrm{H} 3 \mathrm{~K} 27$, which leads to a low expression of AXIN2, ICAT, and GSK3B. AXIN2, ICAT, and GSK3B are negative regulators of the $W N T / \beta$-catenin signaling pathway. NEAT1 inhibits AXIN2, ICAT, and GSK3B and up-regulates WNT/ $\beta$-catenin signaling, eventually promoting glioma cell growth and invasion (66). Zhang et al. found that a lncRNA antisense to FOXM1 (FOXM1-AS) can promote the interaction of ALKBH5 with FOXM1 nascent transcripts, which enhances the demethylation of FOXM1, and leads to the proliferation and tumorigenesis of GSCs (67). Secondly, lncRNAs can act as miRNA sponges. Zhang and colleagues found that the lncRNA TP73-AS1 might compete with HMGB1 for miR142 binding, which acts as a miR-142 sponge and promotes HMGB1 expression. The upregulation of HMGB1 then contributes to glioma cell proliferation and invasion (68). LncRNAs and their regulatory proteins may be drug targets for GBM in the future.

\section{The inevitable difficulty of the BBB}

The BBB refers to the barrier between plasma and brain cells formed by the brain capillary wall and glial cells 
and the barrier between plasma and cerebrospinal fluid formed by the choroid plexus. It blocks the invasion of pathogens to protect the central nervous system (CNS) and maintains the brain environment's stability. However, it also prevents drug delivery in the treatment of GBM, which severely limits the efficacy of drug therapy. Only $5 \%$ of the 7000 drugs in a comprehensive medical chemistry database were able to pass through the BBB (69). The major reasons for drug blockade by the $\mathrm{BBB}$ are tight junctions and adhesion among the monolayer endothelial cells. The BBB is composed of three parts: (I) monolayer endothelial cells that encapsulate capillaries, tight junctions, and adhesion among adjacent endothelial cells; (II) basal layers surrounding the endothelial cells; (III) pericytes and astrocytes, which are directly connected to the basal layers via the synapses (70). There are three ways to cross the BBB: (I) free, passive diffusion of small lipid molecules; (II) vector-mediated transporters; and (III) vesicle-mediated transcellular effects. Small molecules which pass through the BBB by passive diffusion need two conditions: (I) a molecular weight of less than $400 \mathrm{Da}$ and (II) no more than 8 hydrogen bonds (71). The transporters include glucose transporter type 1(GLUT1), which transports mannose, galactose, and glucose; large neutral amino-acid transporter type 1 (LAT1), which transports 10 other large neutral amino acids; cationic amino-acid transporter type 1 (CAT1), which can also carry other cationic amino acids (lysine and ornithine); monocarboxylic acid transporter Type 1 (MCT1), which transports monocarboxylic acids, such as pyruvic acid, ketone bodies, ethyl acetoacetate, and $\beta$-hydroxybutyrate; and adenosine transporters. Vesicle trafficking is mainly divided into clathrin-dependent and non-dependent categories. The vesicle-mediated trafficking is dependent on receptors expressed in endothelial cells, including insulin receptors, ferritin transporters, insulinlike growth factor receptors, leptin receptors, and receptors for some polypeptides.

At present, the main way that drugs pass through the $\mathrm{BBB}$ is via nanoparticles (NPs). NPs are colloidal carriers with a size range of $1-1,000 \mathrm{~nm}$. The nanometer carrier used for drug loading has to meet the following conditions: (I) non-toxic, biodegradable and biocompatible; (II) particle size $<100 \mathrm{~nm}$; (III) stable in blood and not aggregated; (IV) evasive to phagocytes; (V) able to cross the BBB (through receptor-mediated vesicle trafficking); (VI) capable of loading small molecules, polypeptides, proteins, or nucleotides; (VII) minimal changes to drugs (chemical degradation, structural changes, or protein denaturation) due to nanoparticle excipients; (VIII) ability to release the controlled drug; and (IX) economic and efficient production processes (72). Lin et al. developed an albumin-based nanoparticle that can simultaneously load hydrophobic drugs, including paclitaxel and fenretin. Secreted protein acidic and rich in cysteine (SPARC) and glycoprotein 60 (gp60) pass through the BBB to reach the tumor cells (73). Lin et al. also modified albumin nanoparticles by using low molecular weight protamine (LMWP), which helps the nanoparticles penetrate the second barrier formed by the infiltrated tissues. The results from the subcutaneous and orthotopic nude mouse xenograft models showed that the vector could improve the treatment of tumors and reduce toxicity. Also, Xing et al. found that non-viral liposomes could also pass through the BBB (74).

Antibodies pass through the BBB by receptor-mediated vesicle trafficking. The ferferrin receptor (TfR) is the most studied transporter and is highly expressed in the endothelial cells of the BBB. Transferrin (Tf) is a single glycoprotein synthesized in the liver and is the plasma's major ion carrier. After binding to $\mathrm{Fe}^{2+}$, Tf's molecular structure is changed, binding to TfR (75). Anti-TfR antibodies, which were designed based on the Tf-TfR pathway, can significantly improve these antibodies' ability to cross the BBB in rodents and non-human primates (76). However, these antibodies are not sufficiently safe. By proteomic analysis of brain endothelial cells (BEVs) isolated from the rat brain, Zuchero et al. found that bazigin, Glut1, and CD98hc are highly expressed in BEVs (77). Antibodies that target these proteins significantly increase in the brain after their administration, especially antibodies that target CD98hc. Moreover, anti-CD98hc was found to have a significant pharmaceutical response in brain $\mathrm{Ab}$ reduction. Therefore, CD98hc is expected to become a new way for antibodies to enter the brain.

In addition to the above methods, researchers have also tried to promote the passage of drugs through the BBB by using physical methods. By injecting chemicals, such as mannitol, arabinose, urea, fructose, milk amide, and glycerin, capillary osmotic pressure is increased, and this method can cause dehydration of endothelial vascular cells in the brain, which leads to collapse and destruction of tight junctions and promotes the entry of drugs into the brain (72). However, this method causes irreversible damage to the CNS. A pulse-based technique, which relies primarily on the combination of systemic administration and soundactivated microbubbles to non-invasively, transiently, and reversibly open the $\mathrm{BBB}$, allows drug molecules to enter the 
cortex of the brain by crossing the BBB. Carpentier et al. reported an interim result of a clinical trial of a systemic injection of microbubbles with pulsed carboplatin after implantation into a pulsed device system (SonoCloud) in patients with recurrent GBM. Contrast-enhanced MRI showed that the BBB was destroyed when the pulse volume was raised to $1.1 \mathrm{Mpa}$, which did not cause severe side effects in MRI or clinical tests (78). This means that repeated activation of the BBB by a pulse system combined with a systemic microbubble injection is safe and tolerable for patients with recurrent GBM, providing new hope for optimizing the chemotherapeutic drug delivery system.

\section{Conclusions and further perspectives}

The treatment of GBM is a difficult and challenging task. In the past decade, although treatment of GBM has not shown significant improvements, discoveries and new strategies are emerging. The success of immunotherapy in other cancers, together with studies on the formation and mechanisms of cancer stem cells, and more explorations in overcoming the problem of crossing the $\mathrm{BBB}$, may eventually bring new hope to the treatment of GBM.

\section{Acknowledgments}

Funding: This work was supported in part by National Natural Science Foundation of China (81803584 for Qin Tang), Beijing Natural Science Foundation (7212157) and supported by National Key Research and Development Plan (2016YFC1000905). This work was also supported by CAMS Innovation Fund for Medical Sciences (2016-I2M3-007) and Technology Major Projects for "Major New Drugs Innovation and Development" (2018ZX09711001005-025).

\section{Footnote}

Reporting Checklist: The authors have completed the Narrative Review reporting checklist. Available at http:// dx.doi.org/10.21037/atm-20-8017

Conflicts of Interest: All authors have completed the ICMJE uniform disclosure form (available at http://dx.doi. org/10.21037/atm-20-8017). The authors have no conflicts of interest to declare.

Ethical Statement: The authors are accountable for all aspects of the work in ensuring that questions related to the accuracy or integrity of any part of the work are appropriately investigated and resolved.

Open Access Statement: This is an Open Access article distributed in accordance with the Creative Commons Attribution-NonCommercial-NoDerivs 4.0 International License (CC BY-NC-ND 4.0), which permits the noncommercial replication and distribution of the article with the strict proviso that no changes or edits are made and the original work is properly cited (including links to both the formal publication through the relevant DOI and the license). See: https://creativecommons.org/licenses/by-nc-nd/4.0/.

\section{References}

1. Wen PY, Reardon DA, et al. Neuro-oncology in 2015: Progress in glioma diagnosis, classification and treatment. Nat Rev Neurol 2016;12:69-70.

2. Ostrom QT, Gittleman H, Fulop J, et al. CBTRUS Statistical Report: Primary Brain and Central Nervous System Tumors Diagnosed in the United States in 20082012. Neuro Oncol 2015;17 Suppl 4:iv1-iv62.

3. Bähr $\mathrm{O}$, Herrlinger $\mathrm{U}$, Weller $\mathrm{M}$, et al. Very late relapses in glioblastoma long-term survivors. J Neurol 2009;256:1756-8.

4. Furnari FB, Fenton T, Bachoo RM, et al. Malignant astrocytic glioma: genetics, biology, and paths to treatment. Genes Dev 2007;21:2683-710.

5. Simon M, Hosen I, Gousias K, et al. TERT promoter mutations: a novel independent prognostic factor in primary glioblastomas. Neuro Oncol 2015;17:45-52.

6. Turcan S, Rohle D, Goenka A, et al. IDH1 mutation is sufficient to establish the glioma hypermethylator phenotype. Nature 2012;483:479-83.

7. Stupp R, Mason WP, van den Bent MJ, et al. Radiotherapy plus concomitant and adjuvant temozolomide for glioblastoma. N Engl J Med 2005;352:987-96.

8. Newlands ES, Stevens MF, Wedge SR, et al. Temozolomide: a review of its discovery, chemical properties, pre-clinical development and clinical trials. Cancer Treat Rev 1997;23:35-61.

9. Tsang LL, Farmer PB, Gescher A, et al. Characterisation of urinary metabolites of temozolomide in humans and mice and evaluation of their cytotoxicity. Cancer Chemother Pharmacol 1990;26:429-36.

10. Denny BJ, Wheelhouse RT, Stevens MF, et al. NMR and molecular modeling investigation of the mechanism of 
activation of the antitumor drug temozolomide and its interaction with DNA. Biochemistry 1994;33:9045-51.

11. Mojas N, Lopes M, Jiricny J, et al. Mismatch repairdependent processing of methylation damage gives rise to persistent single-stranded gaps in newly replicated DNA. Genes Dev 2007;21:3342-55.

12. Rolhion C, Penault-Llorca F, Kemeny JL, et al. O(6)methylguanine-DNA methyltransferase gene (MGMT) expression in human glioblastomas in relation to patient characteristics and p53 accumulation. Int J Cancer 1999;84:416-20.

13. Zhang J, Stevens MF, Bradshaw TD, et al. Temozolomide: mechanisms of action, repair and resistance. Curr Mol Pharmacol 2012;5:102-14.

14. Pegg AE, Dolan ME, Moschel RC, et al. Structure, function, and inhibition of O6-alkylguanine-DNA alkyltransferase. Prog Nucleic Acid Res Mol Biol 1995;51:167-223.

15. Quinn JA, Jiang SX, Reardon DA, et al. Phase II trial of temozolomide plus o6-benzylguanine in adults with recurrent, temozolomide-resistant malignant glioma. J Clin Oncol 2009;27:1262-7.

16. Friedman HS, Kokkinakis DM, Pluda J, et al. Phase I trial of O6-benzylguanine for patients undergoing surgery for malignant glioma. J Clin Oncol 1998;16:3570-5.

17. Sabharwal A, Middleton MR, et al. Exploiting the role of O6-methylguanine-DNA-methyltransferase (MGMT) in cancer therapy. Curr Opin Pharmacol 2006;6:355-63.

18. Tubbs JL, Pegg AE, Tainer JA, et al. DNA binding, nucleotide flipping, and the helix-turn-helix motif in base repair by O6-alkylguanine-DNA alkyltransferase and its implications for cancer chemotherapy. DNA Repair (Amst) 2007;6:1100-15.

19. Kim SS, Rait A, Kim E, et al. A tumor-targeting p53 nanodelivery system limits chemoresistance to temozolomide prolonging survival in a mouse model of glioblastoma multiforme. Nanomedicine 2015;11:301-11.

20. Gerson SL. Clinical relevance of MGMT in the treatment of cancer. J Clin Oncol 2002;20:2388-99.

21. Lai A, Tran A, Nghiemphu PL, et al. Phase II study of bevacizumab plus temozolomide during and after radiation therapy for patients with newly diagnosed glioblastoma multiforme. J Clin Oncol 2011;29:142-8.

22. Stupp R, Taillibert S, Kanner AA, et al. Maintenance Therapy With Tumor-Treating Fields Plus Temozolomide vs Temozolomide Alone for Glioblastoma: A Randomized Clinical Trial. JAMA 2015;314:2535-43.

23. Jain RK, di Tomaso E, Duda DG, et al. Angiogenesis in brain tumours. Nat Rev Neurosci 2007;8:610-22.

24. Gilbert MR, Dignam JJ, Armstrong TS, et al. A randomized trial of bevacizumab for newly diagnosed glioblastoma. N Engl J Med 2014;370:699-708.

25. Vredenburgh JJ, Desjardins A, Herndon JE 2nd, et al. Phase II trial of bevacizumab and irinotecan in recurrent malignant glioma. Clin Cancer Res 2007;13:1253-9.

26. Hasselbalch B, Lassen U, Hansen S, et al. Cetuximab, bevacizumab, and irinotecan for patients with primary glioblastoma and progression after radiation therapy and temozolomide: a phase II trial. Neuro Oncol 2010;12:508-16.

27. Reardon DA, Desjardins A, Peters KB, et al. Phase II study of carboplatin, irinotecan, and bevacizumab for bevacizumab naïve, recurrent glioblastoma. J Neurooncol 2012;107:155-64.

28. Galanis E, Anderson SK, Lafky JM, et al. Phase II study of bevacizumab in combination with sorafenib in recurrent glioblastoma (N0776): a north central cancer treatment group trial. Clin Cancer Res 2013;19:4816-23.

29. Soffietti R, Trevisan E, Bertero L, et al. Bevacizumab and fotemustine for recurrent glioblastoma: a phase II study of AINO (Italian Association of Neuro-Oncology). J Neurooncol 2014;116:533-41.

30. Taal W, Oosterkamp HM, Walenkamp AM, et al. Singleagent bevacizumab or lomustine versus a combination of bevacizumab plus lomustine in patients with recurrent glioblastoma (BELOB trial): a randomised controlled phase 2 trial. Lancet Oncol 2014;15:943-53.

31. Wick W, Gorlia T, Bendszus M, et al. Lomustine and Bevacizumab in Progressive Glioblastoma. N Engl J Med 2017;377:1954-63.

32. Kamran N, Calinescu A, Candolfi M, et al. Recent advances and future of immunotherapy for glioblastoma. Expert Opin Biol Ther 2016;16:1245-64.

33. Mukasa A, Wykosky J, Ligon KL, et al. Mutant EGFR is required for maintenance of glioma growth in vivo, and its ablation leads to escape from receptor dependence. Proc Natl Acad Sci U S A 2010;107:2616-21.

34. Johnson LA, Scholler J, Ohkuri T, et al. Rational development and characterization of humanized antiEGFR variant III chimeric antigen receptor T cells for glioblastoma. Sci Transl Med 2015;7:275ra22.

35. Babu R, Adamson DC, et al. Rindopepimut: an evidence-based review of its therapeutic potential in the treatment of EGFRvIII-positive glioblastoma. Core Evid 2012;7:93-103.

36. Swartz AM, Li QJ, Sampson JH, et al. Rindopepimut: 
a promising immunotherapeutic for the treatment of glioblastoma multiforme. Immunotherapy 2014;6:679-90.

37. Weller M, Butowski N, Tran DD, et al. Rindopepimut with temozolomide for patients with newly diagnosed, EGFRvIII-expressing glioblastoma (ACT IV): a randomised, double-blind, international phase 3 trial. Lancet Oncol 2017;18:1373-85.

38. Sampson JH, Choi BD, Sanchez-Perez L, et al. EGFRvIII mCAR-modified T-cell therapy cures mice with established intracerebral glioma and generates host immunity against tumor-antigen loss. Clin Cancer Res 2014;20:972-84.

39. Morgan RA, Johnson LA, Davis JL, et al. Recognition of glioma stem cells by genetically modified $\mathrm{T}$ cells targeting EGFRvIII and development of adoptive cell therapy for glioma. Hum Gene Ther 2012;23:1043-53.

40. Debinski W, Gibo DM, Hulet SW, et al. Receptor for interleukin 13 is a marker and therapeutic target for human high-grade gliomas. Clin Cancer Res 1999;5:985-90.

41. Kawakami M, Kawakami K, Takahashi S, et al. Analysis of interleukin-13 receptor alpha2 expression in human pediatric brain tumors. Cancer 2004;101:1036-42.

42. Joshi BH, Puri RA, Leland P, et al. Identification of interleukin-13 receptor alpha2 chain overexpression in situ in high-grade diffusely infiltrative pediatric brainstem glioma. Neuro Oncol 2008;10:265-74.

43. Brown CE, Warden CD, Starr R, et al. Glioma IL13R $\alpha 2$ is associated with mesenchymal signature gene expression and poor patient prognosis. PLoS One 2013;8:e77769.

44. Brown CE, Badie B, Barish ME, et al. Bioactivity and Safety of IL13Ra2-Redirected Chimeric Antigen Receptor CD8+ T Cells in Patients with Recurrent Glioblastoma. Clin Cancer Res 2015;21:4062-72.

45. Brown CE, Alizadeh D, Starr R, et al. Regression of Glioblastoma after Chimeric Antigen Receptor T-Cell Therapy. N Engl J Med 2016;375:2561-9.

46. Krebs S, Chow KK, Yi Z, et al. T cells redirected to interleukin-13R $\alpha 2$ with interleukin-13 mutein--chimeric antigen receptors have anti-glioma activity but also recognize interleukin-13R $\alpha 1$. Cytotherapy 2014;16:1121-31.

47. Nguyen V, Conyers JM, Zhu D, et al. A novel ligand delivery system to non-invasively visualize and therapeutically exploit the IL $13 \mathrm{R} \alpha 2$ tumor-restricted biomarker. Neuro Oncol 2012;14:1239-53.

48. Bhat KPL, Balasubramaniyan V, Vaillant B, et al. Mesenchymal differentiation mediated by NF- $\mathrm{kB}$ promotes radiation resistance in glioblastoma. Cancer Cell 2013;24:331-46.

49. Mao P, Joshi K, Li J, et al. Mesenchymal glioma stem cells are maintained by activated glycolytic metabolism involving aldehyde dehydrogenase 1A3. Proc Natl Acad Sci U S A 2013;110:8644-9.

50. Cheng P, Wang J, Waghmare I, et al. FOXD1-ALDH1A3 Signaling Is a Determinant for the Self-Renewal and Tumorigenicity of Mesenchymal Glioma Stem Cells. Cancer Res 2016;76:7219-30.

51. Jin X, Kim LJY, Wu Q, et al. Targeting glioma stem cells through combined BMI1 and EZH2 inhibition. Nat Med 2017;23:1352-61.

52. Wee B, Pietras A, Ozawa T, et al. ABCG2 regulates self-renewal and stem cell marker expression but not tumorigenicity or radiation resistance of glioma cells. Sci Rep 2016;6:25956.

53. Chow KH, Park HJ, George J, et al. S100A4 Is a Biomarker and Regulator of Glioma Stem Cells That Is Critical for Mesenchymal Transition in Glioblastoma. Cancer Res 2017;77:5360-73.

54. Persano L, Rampazzo E, Della Puppa A, et al. The threelayer concentric model of glioblastoma: cancer stem cells, microenvironmental regulation, and therapeutic implications. ScientificWorldJournal 2011;11:1829-41.

55. Pistollato F, Abbadi S, Rampazzo E, et al. Intratumoral hypoxic gradient drives stem cells distribution and MGMT expression in glioblastoma. Stem Cells 2010;28:851-62.

56. Soeda A, Park M, Lee D, et al. Hypoxia promotes expansion of the CD133-positive glioma stem cells through activation of HIF-1alpha. Oncogene 2009;28:3949-59.

57. McCord AM, Jamal M, Shankavaram UT, et al.

Physiologic oxygen concentration enhances the stem-like properties of CD133+ human glioblastoma cells in vitro. Mol Cancer Res 2009;7:489-97.

58. Lee G, Auffinger B, Guo D, et al. Dedifferentiation of Glioma Cells to Glioma Stem-like Cells By Therapeutic Stress-induced HIF Signaling in the Recurrent GBM Model. Mol Cancer Ther 2016;15:3064-76.

59. Orimo A, Gupta PB, Sgroi DC, et al. Stromal fibroblasts present in invasive human breast carcinomas promote tumor growth and angiogenesis through elevated SDF-1/ CXCL12 secretion. Cell 2005;121:335-48.

60. Dong J, Grunstein J, Tejada M, et al. VEGF-null cells require PDGFR alpha signaling-mediated stromal fibroblast recruitment for tumorigenesis. EMBO J 2004;23:2800-10.

61. Hu X, Sood AK, Dang CV, et al. The role of long noncoding RNAs in cancer: the dark matter matters. Curr Opin Genet Dev 2018;48:8-15.

62. Pandey GK, Mitra S, Subhash S, et al. The risk-associated 
long noncoding RNA NBAT-1 controls neuroblastoma progression by regulating cell proliferation and neuronal differentiation. Cancer Cell 2014;26:722-37.

63. Yoon JH, Abdelmohsen K, Srikantan S, et al. LincRNA-p21 suppresses target mRNA translation. Mol Cell 2012;47:648-55.

64. Singh R, Gupta SC, Peng WX, et al. Regulation of alternative splicing of Bcl-x by BC200 contributes to breast cancer pathogenesis. Cell Death Dis 2016;7:e2262.

65. Deguchi S, Katsushima K, Hatanaka A, et al. Oncogenic effects of evolutionarily conserved noncoding RNA ECONEXIN on gliomagenesis. Oncogene 2017;36:4629-40.

66. Chen Q, Cai J, Wang Q, et al. Long Noncoding RNA NEAT1, Regulated by the EGFR Pathway, Contributes to Glioblastoma Progression Through the WNT/ $\beta$-Catenin Pathway by Scaffolding EZH2. Clin Cancer Res 2018;24:684-95.

67. Zhang S, Zhao BS, Zhou A, et al. m6A Demethylase ALKBH5 Maintains Tumorigenicity of Glioblastoma Stem-like Cells by Sustaining FOXM1 Expression and Cell Proliferation Program. Cancer Cell 2017;31:591-606.e6.

68. Zhang R, Jin H, Lou F, et al. The Long Non-Coding RNA TP73-AS1 Interacted With miR-142 to Modulate Brain Glioma Growth Through HMGB1/RAGE Pathway. J Cell Biochem 2018;119:3007-16.

69. Pardridge WM. The blood-brain barrier: bottleneck in brain drug development. NeuroRx 2005;2:3-14.

70. Obermeier B, Daneman R, Ransohoff RM, et al.

Cite this article as: Zheng X, Tang Q, Ren L, Liu J, Li W, Fu W, Wang J, Du G. A narrative review of research progress on drug therapies for glioblastoma multiforme. Ann Transl Med 2021;9(11):943. doi: 10.21037/atm-20-8017
Development, maintenance and disruption of the bloodbrain barrier. Nat Med 2013;19:1584-96.

71. Pardridge WM. Drug transport across the blood-brain barrier. J Cereb Blood Flow Metab 2012;32:1959-72.

72. Zhang TT, Li W, Meng G, et al. Strategies for transporting nanoparticles across the blood-brain barrier. Biomater Sci 2016;4:219-29.

73. Lin T, Zhao P, Jiang Y, et al. Blood-Brain-BarrierPenetrating Albumin Nanoparticles for Biomimetic Drug Delivery via Albumin-Binding Protein Pathways for Antiglioma Therapy. ACS Nano 2016;10:9999-10012.

74. Xing Y, Wen CY, Li ST, et al. Non-viral liposomemediated transfer of brain-derived neurotrophic factor across the blood-brain barrier. Neural Regen Res 2016;11:617-22.

75. Widera A, Norouziyan F, Shen WC, et al. Mechanisms of TfR-mediated transcytosis and sorting in epithelial cells and applications toward drug delivery. Adv Drug Deliv Rev 2003;55:1439-66.

76. De Bock M, Van Haver V, Vandenbroucke RE, et al. Into rather unexplored terrain-transcellular transport across the blood-brain barrier. Glia 2016;64:1097-123.

77. Zuchero YJ, Chen X, Bien-Ly N, et al. Discovery of Novel Blood-Brain Barrier Targets to Enhance Brain Uptake of Therapeutic Antibodies. Neuron 2016;89:70-82.

78. Carpentier A, Canney M, Vignot A, et al. Clinical trial of blood-brain barrier disruption by pulsed ultrasound. Sci Transl Med 2016;8:343re2. 